\title{
EP-755
}

\section{Mortality of patients with non-functioning pituitary macroadenoma is significantly elevated: systematic analysis of 546 cases in a tertiary referral centre in the UK}

\author{
Georgia Ntali ${ }^{1}$, Cristina Capatina ${ }^{1}$, Violet Fazal-Sanderson ${ }^{1}$, James V Byrne ${ }^{2}$, Simon Cudlip ${ }^{3}$, John AH Wass ${ }^{1}$, Ashley B Grossman ${ }^{1}$, \\ Niki Karavitaki ${ }^{1,4}$
}

${ }^{1}$ Department of Endocrinology, Oxford Centre for Diabetes, Endocrinology and Metabolism, Churchill Hospital, Oxford, UK; ${ }^{2}$ Department of Neuroradiology, John Radcliffe Hospital, Oxford, UK; ${ }^{3}$ Department of Neurosurgery, John Radcliffe Hospital, Oxford, UK; ${ }^{4}$ Centre for Endocrinology, Diabetes and Metabolism, Institute of Biomedical Research, University of Birmingham, Birmingham, UK

Introduction and Aim

- Data on the mortality of patients with non-functioning pituitary macroadenoma (NFA) are limited. The aim of our study was to assess the mortality of patients with NFA and factors predicting it.

Methods

- All patients presenting to the Department of Endocrinology in Oxford with NFA treated surgically between 1963-2011 were studied.

- Status was recorded as either dead or alive, as of 31 December 2011 and data were retrieved through the National Health Service Central Register and the hospital records.

Results

- 546 patients were identified [males/females 333/213; median age at surgery 58.7 years (range 16.1-94.2); median follow-up period 8 years (until date of death or if alive, until date the database was frozen) (range 1 month-48.5 years)].

- During the follow-up period, 83 patients died.

\begin{tabular}{|c|c|c|c|c|c|c|}
\hline Causes of death & $\%$ & & \multirow{2}{*}{$\begin{array}{c}\text { Observed } \\
\text { deaths }\end{array}$} & \multirow{2}{*}{$\begin{array}{c}\text { Expected } \\
\text { deaths }\end{array}$} & \multirow{2}{*}{$\operatorname{SMR}(95 \% \mathrm{CI})$} & \multirow{2}{*}{$p$ value } \\
\hline Cardio/cerebrovascular & 32.5 & & & & & \\
\hline Infections & 30.1 & Total group & 83 & 22.9 & $3.6(2.9-4.5)$ & $<0.001$ \\
\hline Peri-operatively during pituitary surgery & 1.2 & \multirow{2}{*}{$\begin{array}{l}\text { Patients } \\
\text { operated on } \\
\text { before } 1990\end{array}$} & \multirow[t]{2}{*}{14} & \multirow[t]{2}{*}{3} & \multirow[t]{2}{*}{$4.7(2.7-7.6)$} & \multirow[t]{2}{*}{$<0.001$} \\
\hline Gastrointestinal hemorrhage & 1.2 & & & & & \\
\hline Old age & 1.2 & $\begin{array}{l}\text { Patients } \\
\text { operated on } \\
\text { after } 1990\end{array}$ & 69 & 19.6 & $3.5(2.8-4.4)$ & $<0.001$ \\
\hline
\end{tabular}

- Clinical/imaging follow-up data (until date of death or if alive, until date the database was frozen) were available for 436 patients.

\begin{tabular}{|ccc|c|c|c|c|c|}
\hline $\begin{array}{c}\text { Patients } \\
\text { (n) }\end{array}$ & $\begin{array}{c}\text { Males/ } \\
\text { Females } \\
\text { (n) }\end{array}$ & $\begin{array}{c}\text { Median } \\
\text { at surgery } \\
\text { (years) } \\
\text { (range) }\end{array}$ & $\begin{array}{c}\text { No or intrasellar } \\
\text { remnant } \\
\text { post-operatively }\end{array}$ & $\begin{array}{c}\text { Extrasellar } \\
\text { remnant } \\
\text { post-operatively }\end{array}$ & $\begin{array}{c}\text { Details of patients with clinical/imaging follow-up data } \\
\text { follow-up period } \\
\text { (years) } \\
\text { (range) }\end{array}$ & $\begin{array}{c}\text { Regrowth of } \\
\text { NFA }\end{array}$ & $\begin{array}{c}\text { Radiotherapy after } \\
\text { primary surgery or for } \\
\text { regrowth }\end{array}$ \\
\hline 436 & $269 / 167$ & $\begin{array}{c}58.5 \\
(16.1-94.2)\end{array}$ & $203 / 431$ & $228 / 431$ & 6.9 & $111 / 436$ \\
$(1$ month-48.5 years)
\end{tabular}

- Cox regression analysis (univariate approach) demonstrated that amongst age at surgery, NFA regrowth, radiotherapy, sex and extent of removal, the first three were significant predictors of mortality; after multivariate analysis using these three parameters, only age remained an independent significant factor (HR 1.099, 95\% CI:1.073-1.126; $p<0.001$ ).

\section{Conclusions}

- Despite the advances in the management of patients with NFA in the last three decades, mortality remains high.

- Apart from age, factors related with the management/outcome of the tumour including radiotherapy and recurrence are not independent predictors of mortality.

\section{References}

Brada et al. Cerebrovascular mortality in patients with pituitary adenoma. Clin Endocrinol(Oxf) $2002 ; 57: 713-717$.

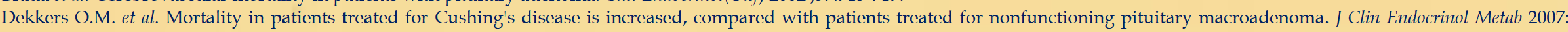
92(3):976-81.

Lindholm J. et al. Hypopituitarism and mortality in pituitary adenoma. Clin Endocrinol(Oxf) 2006 ;65:51-8.

Nielsen E.H. et al. Nonfunctioning pituitary adenoma: incidence, causes of death and quality of life in relation to pituitary function. Pituitary 2007;10(1):67-73. 